\title{
3-Connected Line Graphs of Triangular Graphs are Panconnected and 1-Hamiltonian
}

H. J. Broersma

H. J. Veldman

TWENTE UNIVERSITY OF TECHNOLOGY DEPARTMENT OF APPLIED MATHEMATICS

P.O. BOX 217

7500 AE ENSCHEDE

THE NETHERLANDS

\section{ABSTRACT}

A graph is $k$-triangular if each edge is in at least $k$ triangles. Triangular is a synonym for 1-triangular. It is shown that the line graph of a triangular graph of order at least 4 is panconnected if and only if it is 3-connected. Furthermore, the line graph of a $k$-triangular graph is $k$-hamiltonian if and only if it is $(k+2)$-connected $(k \geq 1)$. These results generalize work of Clark and Wormald and of Lesniak-Foster. Related results are due to Oberly and Sumner and to Kanetkar and Rao.

\section{PRELIMINARIES}

We use [1] for basic terminology and notation, and consider simple graphs only. Let $G$ be a graph. We will often identify a trail in $G$ with the subgraph induced by its edges. Hence a subgraph $T$ of $G$ is a trail if and only if $T$ is connected and at most two vertices of $T$ have odd degree in $T$. An edge $e$ of $G$ is dominated by the trail $T$ if $e$ is incident with at least one vertex of $T ; B(T)$ denotes the set of edges of $G$ dominated by $T$ and we write $b(T)$ for $|B(T)|$. A dominating trail or D-trail of $G$ is a trail that dominates all edges of $G$, while a spanning trail or S-trail contains all vertices of $G$. A circuit is a nontrivial 
closed trail. In particular, an S-circuit is the same as a spanning eulerian subgraph; in the literature, graphs with an S-circuit are often called supereulerian. We will speak of line graphs instead of edge graphs; the line graph of $G$ is denoted $L(G) . G$ is $k$-triangular if every edge of $G$ is contained in at least $k$ triangles ( $k \geq 1) ; G$ is triangular if $G$ is 1-triangular. $G$ is panconnected if $G$ is connected and, for every pair $(u, v)$ of distinct vertices of $G$, there exists a $(u, v)$-path of length $k$ for each $k$ with $d(u, v) \leq k \leq|V(G)|-1 . G$ is $k$-hamiltonian if $G-U$ is hamiltonian for every subset $U$ of $V(G)$ with $0 \leq|U| \leq k$ $(k \geq 0)$. $G$ is locally connected (locally $k$-connected) if, for each vertex $v$ of $G$, the neighborhood $N(v)$ induces a connected (k-connected) subgraph $(k \geq 1)$.

The following characterization of hamiltonian line graphs was obtained in [4].

Theorem 1. (Harary and Nash-Williams [4]). The line graph $L(G)$ of a graph $G$ is hamiltonian if and only if either $G$ has a D-circuit or $G$ is isomorphic to $K_{1,5}$ for some $s \geq 3$.

The following lemmas, including those stated without proof, are easily established.

Lemma 2. Let $e$ and $f$ be distinct edges of a graph $G$ and $T$ a trail in $G$ connecting an end of $e$ and an end of $f$ such that $e, f \notin E(T)$. Then $L(G)$ contains an $(e, f)$-path of length $k$ for each $k$ with $|E(T)|+1 \leq k \leq b(T)-1$.

Lemma 3. If $G$ is a connected and $k$-triangular graph, then $L(G)$ is $(k+1)$ connected $(k \geq 1)$.

Proof. If $G$ is connected and $k$-triangular, then $G$ is $(k+1)$-edge-connected and hence $L(G)$ is $(k+1)$-connected.

Lemma 4. If $G$ is a nontrivial connected triangular graph, then $G$ contains an $S$-circuit.

Proof. Assuming the contrary, let $C$ be a circuit of $G$ of maximum order, $u v$ an edge of $G$ with $u \notin V(C)$ and $v \in V(C)$, and $D$ a triangle containing $u v$. Then the circuit $C^{\prime}$ with $E\left(C^{\prime}\right)=[E(C) \cup E(D)]-[E(C) \cap E(D)]$ contradicts the choice of $C$.

Lemma 5. If $G$ is a $k$-triangular graph, then $L(G)$ is locally $k$-connected $(k \geq 1)$.

\section{MAIN RESULTS}

Theorem 6. Let $G$ be a triangular graph of order at least 4. Then $L(G)$ is panconnected if and only if $L(G)$ is 3-connected. 
Proof. Let $G$ be a triangular graph with $|V(G)| \geq 4$. It is well known that every panconnected graph of order at least 4 is 3-connected. Hence, if $L(G)$ is panconnected, then, since $|V[L(G)]| \geq 4, L(G)$ is 3-connected.

Conversely, assume $L(G)$ is 3-connected, but not panconnected. Then, by Lemma 2, there exists a pair $(e, f)$ of distinct edges of $G$ and a smallest integer $m$ with $d_{L(G)}(e, f) \leq m \leq|E(G)|-1$ such that

(1) There is no trail $T^{\prime}$ between an end of $e$ and an end of $f$ with $\left|E\left(T^{\prime}\right)\right|+$ $1 \leq m \leq b\left(T^{\prime}\right)-1$ and $e, f \notin E\left(T^{\prime}\right)$.

Obviously, $m>d_{L(G)}(e, f)$ and there is a trail $T$ between an end of $e$ and an end of $f$ with $|E(T)|+1 \leq m-1=b(T)-1$ and $e, f \in E(T)$. We additionally assume that

(2) $T$ has maximum length under the given conditions.

Let $e=u_{1} u_{2}$ and $f=v_{1} v_{2}$, where $u_{1}$ is the origin of $T$ and $v_{1}$ the terminus of $T$. We make a number of observations.

(3) If exactly one of the edges $e$ and $f$ has both ends on $T$, then no triangle of $G$ contains both $e$ and $f$.

Assuming the contrary to (3), suppose, e.g., there is a triangle $D$ of $G$ with $V(D)=\left\{u_{1}, u_{2}, v_{2}\right\}$, where $u_{1}, u_{2} \in V(T), v_{2} \notin V(T)$ and $v_{1}=u_{i}$ for $i=1$ or $i=2$. If $v_{2}$ is incident with an edge in $E(G)-B(T)$, then the trail $T^{\prime}$ with $E\left(T^{\prime}\right)=E(T) \cup\left\{u_{3-i} v_{2}\right\}$ contradicts (1), while otherwise $T^{\prime}$ contradicts (2).

(4) $G$ has no triangle $D$ containing at most one of the edges $e$ and $f$ and satisfying $V(D) \cap V(T) \neq \emptyset$ and $E(D) \cap E(T)=\emptyset$.

Assuming the contrary to (4), the trail $T^{\prime}$ with $E\left(T^{\prime}\right)=[E(T) \cup E(D)]$ $-[E(D) \cap\{e, f\}]$ contradicts (1) or (2).

(5) $G$ has no triangle containing neither of the edges $e$ and $f$ and exactly one edge of $T$.

Assuming the contrary to (5), let $D$ be a triangle of $G$ with $E(D) \cap\{e, f\}=\emptyset$ and $E(D) \cap E(T)=\{g\}$. Then the trail $T^{\prime}$ with $E\left(T^{\prime}\right)=(E(T) \cup E(D))-\{g\}$ contradicts (1) or (2).

(6) If some triangle of $G$ contains

(a) a vertex incident with an edge in $E(G)-B(T)$, and

(b) an edge $g$ of $T$, and

(c) exactly one of the edges $e$ and $f$,

then $g$ is a cut-edge of $T$. 
Assuming the contrary to (6), suppose, e.g., there is a triangle $D$ of $G$ with $V(D)=\left\{u_{1}, u_{2}, u\right\}$, where $u_{2}$ is incident with an edge in $E(G)-B(T)$, $u_{1} u \in E(T), u_{2} u \neq f$ and $u_{1} u$ is not a cut-edge of $T$. Then the trail $T^{\prime}$ with $E\left(T^{\prime}\right)=\left[E(T) \cup\left\{u_{2} u\right\}\right]-\left\{u_{1} u\right\}$ contradicts (1).

(7) $T$ is nontrivial.

Assume that $T$ is trivial, so that $u_{1}=v_{1}$. Let $D$ be a triangle containing $u_{1}$. By (4), $D$ contains both $e$ and $f$, and is hence uniquely determined. It follows that $d\left(u_{1}\right)=2$ and $u_{2} v_{2} \in E(G)$. But then the trail $u_{2} v_{2}$ contradicts (1).

Since $b(T)=m \leq|E(G)|-1, G-V(T)$ contains a nontrivial component $H$. By (7) and the fact that $L(G)$ is 3-connected, the edge cut $[V(H), V(T)]$ contains at least three edges. Let $g$ be an edge in $[V(H), V(T)]$ with $g \notin\{e, f\}$ and $D$ a triangle containing $g$. By (3), $D$ does not contain both $e$ and $f$. Hence, by (4), $D$ contains an edge $h$ of $T$. By (5), therefore, $D$ contains exactly one of the edges $e$ and $f$. Now (6) implies that $h$ is a cut edge of $T$.

Assume, e.g., that $V(D)=\left\{u_{1}, u_{2}, u\right\}$, so that $g=u_{2} u$ and $h=u_{1} u$. In particular, $e$ has exactly one end on $T$. We make another observation.

(8) $f$ has exactly one end on $T$.

Assuming that $f$ has both ends on $T,[V(H), V(T)]$ contains an edge $g^{\prime} \notin\{\underline{m}, g\}$. Let $D^{\prime}$ be a triangle containing $g^{\prime}$. Just like $D, D^{\prime}$ contains $e$ and an edge of $T$, $h^{\prime}$ say, which is a cut-edge of $T$. But then the origin $u_{1}$ of $T$ is incident with two cut-edges of $T$, which is impossible.

Let $T_{1}$ be the component of $T-h$ containing $u_{1}$ and let $T_{2}$ be the other component of $T-h$, so that the unique vertex of $T$ incident with $f$ is in $T_{2}$. We make one more observation.

(9) There exists no edge $v u_{1}$ with $v \in V(G)-\left[V(T) \cup\left\{u_{2}\right\}\right]$.

Assuming the contrary to (9), let $D^{\prime}$ be a triangle containing $v u_{1}$. By (4), $D^{\prime}$ contains an edge of $T$. Hence $e \notin E\left(D^{\prime}\right)$ and, by (5), $f \in E\left(D^{\prime}\right)$. It follows that $V\left(D^{\prime}\right)=\left\{v, u_{1}, u\right\}$ and $f=u v$. But then the trail $T^{\prime}$ with $E\left(T^{\prime}\right)=E(T)$ $\cup\left\{u_{2} u, u_{1} v\right\}$ contradicts (1).

$T_{1}$ cannot be trivial, otherwise, in view of (9), the trail $T^{\prime}$ with $E\left(T^{\prime}\right)=$ $\left[E(T) \cup\left\{u_{2} u\right\}\right]-\left\{u_{1} u\right\}$ contradicts $(1)$. Hence $T_{1}$ is a circuit. Since $\left|V\left(T_{1}\right)\right| \neq$ $1 \neq\left|\overline{V\left(T_{1}\right)}\right|$ and $L(G)$ is 3-connected, $\left|\left[V\left(T_{1}\right), \overline{V\left(T_{1}\right)}\right]\right| \geq 3$. Hence $\left.\left[V\left(T_{1}\right), \overline{V\left(T_{1}\right.}\right)\right]$ contains an edge $x y$ with $x \in V\left(T_{1}\right)$ and $x y \notin\left\{u_{1} u, u_{1} u_{2}\right\}$. Since $h$ is a cut-edge of $T, x y \notin E(T)$. Let $D^{\prime}$ be a triangle containing $x y$. We now establish the theorem by deriving contradictions in all possible cases.

Case 1. $x \neq u_{1}$.

Then $x$ is incident with neither of the edges $e$ and $f$, so that $D^{\prime}$ contains at most one of the edges $e$ and $f$. By (4) and (8), $f \notin E\left(D^{\prime}\right)$. Two possibilities remain. 
Case 1.1. $e \in E\left(D^{\prime}\right)$.

Then $V\left(D^{\prime}\right)=\left\{x, u_{1}, u_{2}\right\}$ and, by (4), $x u_{1} \in E\left(T_{1}\right)$. (6) now implies that $x u_{1}$ is a cut-edge of $T$, contradicting the fact that $T_{1}$, being a circuit, is 2-edgeconnected.

Case 1.2. $e \notin E\left(D^{\prime}\right)$.

Then, by (4) and (5), $\left|E\left(D^{\prime}\right) \cap E(T)\right|=2$. It follows that $V\left(D^{\prime}\right)=\left\{x, u_{1}, u\right\}$ and $x u_{1} \in E\left(T_{1}\right)$. Now the trail $T^{\prime}$ with $E\left(T^{\prime}\right)=\left(E(T) \cup\left\{u_{2} u, u x\right\}\right)-\left\{x u_{1}\right\}$ contradicts (1).

Case 2. $x=u_{1}$.

Then, by (9), $y \in V\left(T_{2}\right)$. By (4) and (8), it is impossible that $D^{\prime}$ contains exactly one of the edges $e$ and $f$. Two possibilities remain.

Case 2.1. $D^{\prime}$ contains both $e$ and $f$.

Then $V\left(D^{\prime}\right)=\left\{u_{1}, u_{2}, y\right\}$ and $f=y u_{2}$. Now the trail $T^{\prime}$ with $E\left(T^{\prime}\right)=\mid E(T) \cup$ $\left.\left\{u_{2} u, y u_{1}\right\}\right]-\left\{u u_{1}\right\}$ contradicts (1).

Case 2.2. $D^{\prime}$ contains neither $e$ nor $f$.

Then, by (4) and (5), $\left|E\left(D^{\prime}\right) \cap E(T)\right|=2$. It follows that $V\left(D^{\prime}\right)=\left\{u_{1}, u, y\right\}$ and $u y \in E\left(T_{2}\right)$. Now the trail $T^{\prime}$ with $E\left(T^{\prime}\right)=\left[E(T) \cup\left\{u_{2} u, u_{1} y\right\}\right]-\{u y\}$ contradicts (1).

Corollary 7. (Clark and Wormald $[3]$ ). If $G$ is a connected 2-triangular graph, then $L(G)$ is panconnected.

Proof Combine Lemma 3 and Theorem 6.

Corollary 8. (Clark and Wormald [3]). If $G$ is a 2-connected triangular graph, then $L(G)$ is panconnected.

Proof. Let $[S, \bar{S}]$ be an edge-cut of $G$ with $|S| \neq 1 \neq|\bar{S}|$. Since $G$ is 2connected, $[S, \bar{S}]$ contains two nonadjacent edges. Since $G$ is triangular, $[S, \bar{S}]$ must contain a third edge. It follows that $L(G)$ is 3 -connected and we are done by Theorem 6 .

Let $G$ be a connected graph with $\delta(G) \geq 3$. Then $L(G)$ is triangular. Furthermore, it is easily shown that $L[L(G)]$ is 3 -connected if and only if $G$ has no cut vertex of degree 3 (cf. [6]). Thus Theorem 6 has the following consequence also: 
Corollary 9. Let $G$ be a connected graph with $\delta(G) \geq 3$. Then $L[L(G)]$ is panconnected if and only if $G$ contains no cut vertex of degree 3.

The next result shows that, in Theorem 6, "panconnected" may be replaced by "1-hamiltonian."

Theorem 10. Let $G$ be a $k$-triangular graph $(k \geq 1)$. Then $L(G)$ is $k$-hamiltonian if and only if $L(G)$ is $(k+2)$-connected.

Proof. It suffices to prove the theorem for $k=1$; the proof is then completed by induction on $k$, using Theorem 1 and Lemma 4 . Since 1-hamiltonian graphs are necessarily 3 -connected, it remains to establish sufficiency.

Let $G$ be a triangular graph and assume $L(G)$ is 3-connected, but not 1hamiltonian. By Theorem 1 and Lemma $4, L(G)$ is hamiltonian, so there is a vertex $e$ of $L(G)$ such that $L(G)-e$ is nonhamiltonian. $e$ is an edge of $G$ and, by Theorem 1, $G-e$ has no D-circuit. Let $C$ be a circuit of $G-e$ such that

(10) $C$ has maximum order among all circuits of $G-e$.

We make the following observation:

(11) $G-e$ has no cycle $C_{0}$ satisfying $V\left(C_{0}\right) \cap V(C) \neq \emptyset \neq V\left(C_{0}\right) \cap[V(G)-V(C)]$ and $\left|E\left(C_{0}\right) \cap E(C)\right| \leq 1$.

Assuming the contrary to (11), the circuit $C^{\prime}$ with $E\left(C^{\prime}\right)=\left[E(C) \cup E\left(C_{0}\right)\right]$ - $\left[E(C) \cap E\left(C_{0}\right)\right]$ contradicts (10).

Since $C$ is not a D-circuit, $G-V(C)$ has a nontrivial component $H$. Since $L(G)$ is 3-connected, $|[V(H), V(C)]| \geq 3$. Hence there exist two distinct triangles $D_{1}$ and $D_{2}$ with $E\left(D_{i}\right) \cap[V(H), V(C)] \neq \emptyset(i=1,2)$. By (11), both $D_{1}$ and $D_{2}$ contain $e$. $\left(D_{1} \cup D_{2}\right)-e$ is a cycle of length 4 , which, by (11). has two edges in common with $C$. It follows that $e$ has exactly one end on $C, v$ say. Let $u$ be the other end of $e$ and $v_{i}$ the unique vertex in $V\left(D_{i}\right)-\{u, v\}$, so that $v v_{i} \in E(C)(i=1,2)$.

We now show that $D_{1}$ and $D_{2}$ are the only triangles of $G$ containing edges of $D_{1} \cup D_{2}$. It then follows that every nontrivial component of $G-$ $\left[E\left(D_{1}\right) \cup E\left(D_{2}\right)\right]$ is triangular and hence, by Lemma 4 , contains an S-circuit. The proof is then completed by noting that the union of $\left(D_{1} \cup D_{2}\right)-e$ with Scircuits of the nontrivial components of $G-\left[E\left(D_{1}\right) \cup E\left(D_{2}\right)\right]$ is an S-circuit of $G-e$, contradicting the fact that not even $G-e$ has a D-circuit.

Suppose $G$ contains a triangle $D$ with $D \neq D_{1}, D_{2}$ and $E(D) \cap\left[E\left(D_{1}\right) \cup\right.$ $\left.E\left(D_{2}\right)\right] \neq \emptyset$. By $(11), D$ contains neither of the edges $u v_{1}$ and $u v_{2}$. We derive contradictions in two cases.

Case 1. $D$ contains $e$. 
Let $v_{3}$ be the vertex in $V(D)-\{u, v\}$. From (11) we deduce that $v v_{3} \in E(C)$. Let $F=\left\{v v_{1}, v v_{2}, v v_{3}\right\}$. $F$ contains two edges $f$ and $g$ such that $C-\{f, g\}$ is a connected subgraph of $G$ : if all edges of $F$ belong to the same block of $C$, then $f$ and $g$ are arbitrarily chosen from $F$, whereas in the opposite case $f$ and $g$ are chosen in different blocks of $C$. If, e.g., $f=v v_{1}$ and $g=v v_{2}$, then it follows that the subgraph $C^{\prime}$ of $G$ with $V\left(C^{\prime}\right)=V(C) \cup\{u\}$ and $E\left(C^{\prime}\right)=[E(C) \cup$ $\left.\left\{u v_{1}, u v_{2}\right\}\right]-\left\{v v_{1}, v v_{2}\right\}$ is a circuit that contradicts $(10)$.

Case 2. D contains $v v_{1}$ or $v v_{2}$.

Suppose, e.g., $D$ contains $v v_{1}$. By (11), $v_{1}$ and $v_{2}$ are nonadjacent, so $D$ contains a vertex $w$ with $w \notin V\left(D_{1}\right) \cup V\left(D_{2}\right)$. Again by (11), at least one of the edges $v w$ and $v_{1} w$ is in $E(C)$. If $v w \in E(C)$ and $v_{1} w \notin E(C)$, then the circuit with edge set $\left(E(C) \cup\left\{u v_{1}, u v_{2}, v_{1} w\right\}\right)-\left\{v v_{2}, v w\right\}$ contradicts (10). If $v w \notin E(C)$ and $v_{1} w \in E(C)$, then the circuit with edge set $[E(C) \cup$ $\left.\left\{u v_{1}, u v_{2}, v w\right\}\right]-\left\{v v_{2}, v_{1} w\right\}$ contradicts (10). Finally, if $v w \in E(C)$ and $v_{1} w \in$ $E(C)$, then (10) is violated by the circuit with edge set $[E(C) \cup$ $\left.\left\{u v_{1}, u v_{2}\right\}\right]-\left\{v v_{1}, v v_{2}\right\}$

Corollary 11. (Lesniak-Foster [6]). Let $G$ be a connected graph with $\delta(G) \geq 3$. Then $L[L(G)]$ is $(\delta(G)-3)$-hamiltonian.

Proof. $L(G)$ is connected and $[\delta(G)-2]$-triangular, so that, by Lemma 3 , $L[L(G)]$ is $[\delta(G)-1]$-connected. The proof is completed by applying Theorem 1 and Lemma 4 in case $\delta(G)=3$ and Theorem 10 in case $\delta(G) \geq 4$.

Note that Theorem 10 also implies that, if $G$ is a connected graph with $\delta(G) \geq 3$ such that $L[L(G)]$ is not $[\delta(G)-2]$-hamiltonian, then $L[L(G)]$ is not $\delta(G)$-connected.

The next consequence of Theorem 10 is completely analogous to Corollary 9.

Corollary 12. (Lesniak-Foster [6]). Let $G$ be a connected graph with $\delta(G) \geq 3$. Then $L[L(G)]$ is 1 -hamiltonian if and only if $G$ contains no cut vertex of degree 3 .

Via a trivial variation on the proof of Theorem 10 we obtain the following result:

Theorem 13. Let $G$ be a $k$-triangular graph $(k \geq 1)$. Then the following statements are equivalent:

(i) $G-F$ contains an S-circuit for every subset $F$ of $E(G)$ with $0 \leq$ $|F| \leq k$;

(ii) $G$ is $(k+2)$-edge-connected. 


\section{RELATED RESULTS AND CONJECTURES}

Oberly and Sumner [7] have shown that every connected, locally connected graph of order at least 3 containing no induced $K_{1.3}$ is hamiltonian. Via induction on $k$ one immediately obtains the following generalization:

Theorem 14. If $G$ is a connected, locally $k$-connected graph of order at least 3 containing no induced $K_{1.3}$, then $G$ is $(k-1)$-hamiltonian $(k \geq 1)$.

In view of Lemma 5 and the fact that no line graph contains an induced $K_{1,3}$, Corollary 11 is a consequence of Theorem 14 , too. Likewise, Corollary 7 is also implied by the following result:

Theorem 15. (Kanetkar and Rao [5]). If $G$ is a connected, locally 2-connected graph containing no induced $K_{1,3}$, then $G$ is panconnected.

By Lemma 5, Theorem 6 is a special case of the following conjectured improvement on Theorem 15:

Conjecture 16. Let $G$ be a connected, locally connected graph of order at least 4 containing no induced $K_{1,3}$. Then $G$ is panconnected if and only if $G$ is 3connected.

The next conjecture is analogous to Conjecture 16 .

Conjecture 17. Let $G$ be a connected, locally $k$-connected graph containing no induced $K_{1.3}(k \geq 1)$. Then $G$ is $k$-hamiltonian if and only if $G$ is $(k+2)$ connected.

Conjecture 17 is more general than Theorem 10. In [2] it was shown that every connected, locally $k$-connected graph is $(k+1)$-connected $(k \geq 1)$. Hence Conjecture 17 also generalizes Theorem 14 for $k \geq 2$. Again, it suffices to prove Conjecture 17 for $k=1$.

Finally, consider the following statement:

(*) Let $G$ be a $k$-triangular graph $(k \geq 1)$. Then $L(G)$ is s-hamiltonian if and only if $L(G)$ is $(s+2)$-connected.

By Theorem $10,\left(^{*}\right)$ is true for $s \leq k$. For given $k$, it would be interesting to know for which values of $s\left({ }^{*}\right)$ holds. If, e.g., it were shown that, for each $k \geq 2,\left(^{*}\right)$ holds for $s=2 k$, then the following result of Lesniak-Foster [6] would be generalized: if $G$ is a 2-connected graph with $\delta(G) \geq 4$, then $L[L(G)]$ is $[2 \delta(G)-4]$-hamiltonian. Similarly, improvements on Conjecture 17 may be possible. 


\section{References}

[1] J. A. Bondy and U. S. R. Murty, Graph Theory with Applications. Macmillan, London, and Elsevier, New York (1976).

[2] G. Chartrand, R. J. Gould, and A. D. Polimeni, A note on locally connected and Hamiltonian-connected graphs. Isr. J. Math. 33 (1979) 5-8.

[3] L. H. Clark and N. C. Wormald, Hamiltonian-like indices of graphs. Ars Combinat. 15 (1983) 131-148.

[4] F. Harary and C. St. J. A. Nash-Williams, On eulerian and hamiltonian graphs and line graphs. Canad. Math. Bull. 8 (1965) 701-710.

[5] S. V. Kanetkar and P. R. Rao, Connected, locally 2-connected, $K_{1.3}$-free graphs are panconnected. J. Graph Theory 8 (1984) 347-353.

[6] L. Lesniak-Foster, On n-hamiltonian line graphs. J. Combin. Theory (B) 22 (1977) 263-273.

[7] D. J. Oberly and D. P. Sumner, Every connected, locally connected nontrivial graph with no induced claw is hamiltonian. J. Graph Theory 3 (1979) 351-356. 